\title{
Radicals and units in Ramanujan's work
}

\author{
by \\ Bruce C. Berndt (Urbana, Ill.), Heng Huat Chan (Singapore) \\ and Liang-Cheng Zhang (Springfield, Mo.)
}

In memory of S. Chowla

1. Introduction. In problems he submitted to the Journal of the Indian Mathematical Society [16], in his notebooks [15], and in his lost notebook [17], Ramanujan established many intriguing equalities between radicals. In particular, in his extensive calculations of more than 100 class invariants, he frequently needed to establish difficult radical equalities; see two papers [3], [5] by the authors dedicated to the calculation of Ramanujan's class invariants, and Berndt's book [2, Chap. 34]. Ramanujan had an uncanny ability in discerning radical equalities, but sometimes we can obtain a peek into Ramanujan's thinking by observing that units play a key role.

In Section 2 of this brief note, we reexamine the radical identities that Ramanujan submitted as problems to the Journal of the Indian Mathematical Society. We will see how units come into play, and we will also put some of the radical equalities in more general settings.

In Section 3, we examine some material in Ramanujan's notebooks [15] and lost notebook [17], mostly pertaining to class invariants and singular moduli. So that we may define the class invariants of Ramanujan and Weber [19], set

$$
\chi(q):=\prod_{n=0}^{\infty}\left(1+q^{2 n+1}\right), \quad|q|<1 .
$$

If $q=\exp (-\pi \sqrt{n})$, where $n$ is a positive rational number, the two class invariants $G_{n}$ and $g_{n}$ are defined by

$$
G_{n}:=2^{-1 / 4} q^{-1 / 24} \chi(q) \text { and } g_{n}:=2^{-1 / 4} q^{-1 / 24} \chi(-q) .
$$

It is customary to study $G_{n}$ for odd $n$ and $g_{n}$ for even $n$. As usual, in the theory of elliptic functions, let $k:=k(q), 0<k<1$, denote the modulus.

1991 Mathematics Subject Classification: Primary 11R27. 
The singular modulus $k_{n}$ is defined by $k_{n}:=k\left(e^{-\pi \sqrt{n}}\right)$, where $n$ is a natural number. After Ramanujan, set $\alpha_{n}=k_{n}^{2}$. Except for possibly a power of 2, $G_{n}, g_{n}$, and $k_{n}$ are units [2, p. 184, Theorem 1.1]. The authors have verified many of Ramanujan's formulas for class invariants and singular moduli [3], $[5],[4],[2],[20]$. These calculations are often very difficult, but with the observation that certain algebraic expressions are units or have the character of units, seemingly very difficult calculations can be transformed into considerably easier ones.

In Section 4, we briefly examine some elementary radical identities found in Ramanujan's lost notebook.

Radicals arise in other problems that Ramanujan submitted to the Journal of the Indian Mathematical Society, and we encourage readers to examine them in Ramanujan's Collected Papers [16, pp. 322-334]. See also Berndt's book [1, Chap. 22] for an assortment of beautiful elementary algebraic identities, many involving radicals.

Lastly, some of the ideas here are briefly touched in our paper [6].

\section{Elementary equalities between radicals}

THEOREM 2.1 .

$$
(\sqrt[3]{5}-\sqrt[3]{4})^{1 / 2}=\frac{1}{3}(\sqrt[3]{2}+\sqrt[3]{20}-\sqrt[3]{25})
$$

$$
(\sqrt[3]{28}-\sqrt[3]{27})^{1 / 2}=\frac{1}{3}(\sqrt[3]{98}-\sqrt[3]{28}-1)
$$

$$
\left(\sqrt[5]{\frac{1}{5}}+\sqrt[5]{\frac{4}{5}}\right)^{1 / 2}=(1+\sqrt[5]{2}+\sqrt[5]{8})^{1 / 5}
$$

$$
\begin{aligned}
& \left(\sqrt[5]{\frac{1}{5}}+\sqrt[5]{\frac{4}{5}}\right)^{1 / 2}=\sqrt[5]{\frac{16}{125}}+\sqrt[5]{\frac{8}{125}}+\sqrt[5]{\frac{2}{125}}-\sqrt[5]{\frac{1}{125}}, \\
& \left(\sqrt[5]{\frac{32}{5}}-\sqrt[5]{\frac{27}{5}}\right)^{1 / 3}=\sqrt[5]{\frac{1}{25}}+\sqrt[5]{\frac{3}{25}}-\sqrt[5]{\frac{9}{25}}
\end{aligned}
$$

$$
\left(\frac{3+2 \sqrt[4]{5}}{3-2 \sqrt[4]{5}}\right)^{1 / 4}=\frac{\sqrt[4]{5}+1}{\sqrt[4]{5}-1}
$$

$$
(7 \sqrt[3]{20}-19)^{1 / 6}=\sqrt[3]{\frac{5}{3}}-\sqrt[3]{\frac{2}{3}},
$$

$$
\left(4 \sqrt[3]{\frac{2}{3}}-5 \sqrt[3]{\frac{1}{3}}\right)^{1 / 8}=\sqrt[3]{\frac{4}{9}}-\sqrt[3]{\frac{2}{9}}+\sqrt[3]{\frac{1}{9}},
$$

$$
(\sqrt[3]{2}-1)^{1 / 3}=\sqrt[3]{\frac{1}{9}}-\sqrt[3]{\frac{2}{9}}+\sqrt[3]{\frac{4}{9}} .
$$

Equalities (a) and (b) constitute Question 525 [11], [16, p. 329]. Equalities (c)-(f) comprise Question 1070 [13], [16, p. 334]. Parts (g) and (h) are found in Question 1076 [14], [16, p. 334]. Lastly, equality (i) is part of Question 682 [12], [16, p. 331].

Each identity can be easily verified by taking an appropriate power of each side above and then simplifying the right side. Both the left and right sides of each equality in Theorem 2.1 are units in some algebraic number field. Although Ramanujan never used the term unit, and probably did 
not formally know what a unit was, he evidently recognized their essential essence. He then recognized that taking certain powers of units often led to elegant identities.

We will briefly explain why these expressions are units. It suffices to examine the left sides. First observe that if $x=\sqrt[3]{a}-\sqrt[3]{a-1}$, then

$$
x^{3}=1-3 \sqrt[3]{a(a-1)} x .
$$

We thus see that $x$ is a unit if $a=t / 3$, for some algebraic integer $t$. Secondly, observe that if $x=\sqrt[5]{a}-\sqrt[5]{a-1}$ and $y=\sqrt[5]{a(a-1)}$, then

$$
x^{5}+5 x^{3} y+5 x y^{2}-1=0 .
$$

Thus, $x$ is a unit if $a=t / 5$, for some algebraic integer $t$. More generally, it is not difficult to check that $\sqrt[n]{1+a}-\sqrt[n]{a}$ is a unit whenever $n$ is a positive integer and $n a$ is an algebraic integer. Similar arguments can be given for other expressions appearing on the left sides above.

For identities (a), (b), (e), (g), and (i) we provide generalizations below. For (c), we establish general analogues.

Proposition 2.2. For $\frac{1}{2}(1-\sqrt{3})^{3} \leq a \leq \frac{1}{2}(1+\sqrt{3})^{3}$,

$$
\left(\frac{(a+4) \sqrt[3]{a}+(1-2 a) \sqrt[3]{4}}{9}\right)^{1 / 2}=\frac{\sqrt[3]{2}+\sqrt[3]{4 a}-\sqrt[3]{a^{2}}}{3} .
$$

Proof. It is easy to verify that

$$
\left(\sqrt[3]{2}+\sqrt[3]{4 a}-\sqrt[3]{a^{2}}\right)^{2}=(a+4) \sqrt[3]{a}+(1-2 a) \sqrt[3]{4}
$$

which is equivalent to (2.1) for the given values of $a$.

If $a$ is a real number lying outside the interval specified in Proposition 2.2, then (2.1) is still valid if the right side is multiplied by -1 .

Setting $a=5$ in (2.1), we deduce (a) above. One can deduce further interesting radical identities by giving $a$ special values in (2.1). For example, setting $a=14$, we find that

$$
(2 \sqrt[3]{14}-3 \sqrt[3]{4})^{1 / 2}=\frac{1}{3}(\sqrt[3]{196}-\sqrt[3]{2}-2 \sqrt[3]{7})
$$

Proposition 2.3. For a outside the interval $\left[\frac{1}{4}(1-\sqrt{3})^{3}, \frac{1}{4}(1+\sqrt{3})^{3}\right]$,

$$
\left(\frac{(a+2) \sqrt[3]{4 a}+(1-4 a)}{9}\right)^{1 / 2}=\frac{\sqrt[3]{2 a^{2}}-\sqrt[3]{4 a}-1}{3} .
$$

Proof. It is easy to verify that

$$
\left(\sqrt[3]{2 a^{2}}-\sqrt[3]{4 a}-1\right)^{2}=(a+2) \sqrt[3]{4 a}+(1-4 a),
$$

from which (2.2) follows for the given values of $a$.

If $a$ lies in the interior of the interval specified in Proposition 2.3, then the right side must be multiplied by -1 . 
Setting $a=7$ in (2.2), we deduce (b) of Theorem 2.1. Other interesting identities can be deduced by specializing (2.2). For example, setting $a=25$ in $(2.2)$, we find that

$$
(3 \sqrt[3]{100}-11)^{1 / 2}=\frac{1}{3}(5 \sqrt[3]{10}-\sqrt[3]{100}-1) .
$$

The proofs of (a) and (b) given by N. S. Aiyar [11] proceed along completely different lines.

Proposition 2.4. For each real number $a$,

$$
\left\{(3 a+1)+(3-a) \sqrt[5]{a}-5 \sqrt[5]{a^{3}}\right\}^{1 / 3}=1+\sqrt[5]{a}-\sqrt[5]{a^{2}} .
$$

P r o of. Raising each side of (2.3) to the third power, we readily verify the truth of $(2.3)$.

Setting $a=3$ in (2.3) and dividing both sides by $\sqrt[5]{25}$, we deduce (e) in Theorem 2.1.

Proposition 2.5. If $a$ is any real number, then

$$
\begin{array}{r}
\left\{(9 a+15) \sqrt[3]{a^{2}(a+1)}+(9 a-6) \sqrt[3]{a(a+1)^{2}}-(18 a(a+1)-1)\right\}^{1 / 6} \\
=\sqrt[3]{a+1}-\sqrt[3]{a} .
\end{array}
$$

P r o of. Taking the sixth power of each side of (2.4) and simplifying, we complete the proof.

If we set $a=2 / 3$ in (2.4), we deduce (g) of Theorem 2.1. Note that if $3 a$ is an algebraic integer in (2.4), we obtain units on each side. Giving $a$ other values in (2.4), we can establish further interesting radical identities. For example, letting $a=1$ and $a=1 / 3$, we deduce that

$$
(24 \sqrt[3]{2}+3 \sqrt[3]{4}-35)^{1 / 6}=\sqrt[3]{2}-1
$$

and

respectively.

$$
(6 \sqrt[3]{4}-2 \sqrt[3]{2}-7)^{1 / 6}=\sqrt[3]{\frac{4}{3}}-\sqrt[3]{\frac{1}{3}}
$$

In both the original formulation of Question 1076 [14] and Ramanujan's Collected Papers [16, p. 334], the exponent $1 / 6$ on the left side of $(\mathrm{g})$ is incorrectly printed as $1 / 8$. In fact, the powers $1 / 6$ and $1 / 8$ are permuted on the left sides of $(\mathrm{g})$ and $(\mathrm{h})$ in both the original statements and the Collected Papers. In contrast to (g), we do not have a generalization of (h). However, we offer a simple proof of $(\mathrm{h})$ below.

Pro of of (h). It is easy to see that (h) is equivalent to the equality

$$
\left(\sqrt[3]{\frac{4}{9}}-\sqrt[3]{\frac{2}{9}}+\sqrt[3]{\frac{1}{9}}\right)^{-8}=\left(4 \sqrt[3]{\frac{2}{3}}-5 \sqrt[3]{\frac{1}{3}}\right)^{-1},
$$

which can be written in the alternative form

$$
\left(\sqrt[3]{\frac{2}{3}}+\sqrt[3]{\frac{1}{3}}\right)^{8}=16 \sqrt[3]{\frac{4}{9}}+20 \sqrt[3]{\frac{2}{9}}+25 \sqrt[3]{\frac{1}{9}},
$$

which can be verified by expanding the left side. 
We next establish a general identity which has (i) as a special case.

Proposition 2.6. For any real number a,

$$
\left(\left(a^{2}-7 a+1\right)+(6 a-3) \sqrt[3]{a}+(6-3 a) \sqrt[3]{a^{2}}\right)^{1 / 3}=\sqrt[3]{a^{2}}-\sqrt[3]{a}+1 .
$$

P r o of. Cubing both sides of (2.6) we readily establish its truth.

As an example, set $a=2$ in (2.6) and divide both sides by $3^{2 / 3}$ to deduce that

which is (i).

$$
(\sqrt[3]{2}-1)^{1 / 3}=\sqrt[3]{\frac{4}{9}}-\sqrt[3]{\frac{2}{9}}+\sqrt[3]{\frac{1}{9}}
$$

We do not have any generalizations of (c) and (d). Identity (c) can be verified by taking the tenth power of each side, expanding both sides, and simplifying. Likewise, (d) can be verified by squaring both sides. S. D. Chowla, N. B. Mitra, and S. V. Venkataraya Sastri established (c) in the same way [13]. However, their proof of (d) is rather ingenious. Let $a, b, c$, and $d$ be numbers satisfying the relations

$$
a^{5}=2 b^{5}=8 c^{5}=16 d^{5} .
$$

Then it is easily proved by squaring that

$$
a+b+c-d=\sqrt{\left(c^{2}+2 a b\right)+\left(d^{2}+2 a c\right)} .
$$

Putting

we deduce (d).

$$
a=\sqrt[5]{\frac{16}{125}}, \quad b=\sqrt[5]{\frac{8}{125}}, \quad c=\sqrt[5]{\frac{2}{125}}, \quad d=\sqrt[5]{\frac{1}{125}},
$$

Although we have no generalization for (c), we have found two new analogues of (c), namely,

$$
\left(\sqrt[3]{\frac{4}{3}}-\sqrt[3]{\frac{1}{3}}\right)^{1 / 2}=(\sqrt[3]{2}-1)^{1 / 3}
$$

and

$$
\left(\sqrt[3]{\frac{16}{9}}+\sqrt[3]{\frac{4}{9}}+\sqrt[3]{\frac{1}{9}}\right)^{1 / 2}=(\sqrt[3]{4}+\sqrt[3]{2}+1)^{1 / 3}
$$

both of which can be readily verified by taking the sixth powers of both sides.

We have nothing to add about (f), which is easily verified.

On page 344 in his lost notebook [17], Ramanujan offers the (corrected) equalities

$$
\sqrt[3]{\frac{1}{3}}+\sqrt[3]{\frac{5}{3}}=\sqrt[6]{\frac{\sqrt[3]{5}-1}{2-\sqrt[3]{5}}}=\sqrt[3]{\frac{3+\sqrt[3]{5}}{\sqrt[3]{5}-1}}=\sqrt[5]{\frac{3 \sqrt[3]{3}+\sqrt[3]{15}}{2-\sqrt[3]{5}}}
$$

The factor $\sqrt[6]{3}$ after the first equality was omitted by Ramanujan. It can be shown that the far left side of (2.7) equals each of the three remaining radicals by taking the square, cube, and fifth powers of each, respectively. 
3. Radicals appearing in the calculation of class invariants, singular moduli, and continued fractions. The authors have devoted much effort in recent years to the calculation of Ramanujan's class invariants. In particular, in [5] 13 class invariants were proved. After our paper was published, we noticed that in 12 of our 13 calculations, we used (sometimes in somewhat altered form) special cases of one of the two identities

$$
\left\{\left(8 a^{2}-1\right)+\sqrt{\left(8 a^{2}-1\right)^{2}-1}\right\}^{1 / 4}=\sqrt{a+\frac{1}{2}}+\sqrt{a-\frac{1}{2}}
$$

and

$$
\left\{\left(32 b^{3}-6 b\right)+\sqrt{\left(32 b^{3}-6 b\right)^{2}-1}\right\}^{1 / 6}=\sqrt{b+\frac{1}{2}}+\sqrt{b-\frac{1}{2}} .
$$

These identities can be verified by raising each side to the fourth and sixth powers, respectively. For example, setting $a=(5+\sqrt{65}) / 8$ in $(3.1)$, we obtain the identity

$$
\left\{\frac{41+5 \sqrt{65}}{4}+\sqrt{\left(\frac{41+5 \sqrt{65}}{4}\right)^{2}-1}\right\}^{1 / 4}=\sqrt{\frac{9+\sqrt{65}}{8}}+\sqrt{\frac{1+\sqrt{65}}{8}},
$$

utilized in the calculation of the invariant $G_{65}$. Setting $b=(4+3 \sqrt{3}) / 4$ in (3.2), we find that

$$
\left\{(188+108 \sqrt{3})+\sqrt{(188+108 \sqrt{3})^{2}-1}\right\}^{1 / 6}=\sqrt{\frac{6+3 \sqrt{3}}{4}}+\sqrt{\frac{2+3 \sqrt{3}}{4}},
$$

used to determine the class invariant $G_{69}$. Some of the determinations in [20] also depend on (3.1) and (3.2).

We are very grateful to Bruce Reznick for informing us that, in fact, (3.1) and (3.2) are special cases of a theorem about Chebyshev polynomials. Recall that the $n$th Chebyshev polynomial $T_{n}(x)$ is defined by $T_{n}(x)=\cos (n \theta)$, where $\theta=\cos ^{-1} x$. We now state and prove Reznick's theorem.

Theorem 3.1. If $x \geq 1 / 2$ and $n$ is a positive integer, then

$$
\left(\sqrt{x+\frac{1}{2}}+\sqrt{x-\frac{1}{2}}\right)^{2 n}=T_{n}(2 x)+\sqrt{T_{n}^{2}(2 x)-1} .
$$

Proof. Put $x=(1 / 2) \cos \theta$, so that $\theta$ is purely imaginary when $x>1 / 2$. Then

$$
\begin{aligned}
\left(\sqrt{x+\frac{1}{2}}+\sqrt{x-\frac{1}{2}}\right)^{2 n}=\left(\sqrt{\frac{1}{2}(1+\cos \theta)}+\sqrt{\frac{1}{2}(\cos \theta-1)}\right)^{2 n} \\
=\left(\cos \left(\frac{1}{2} \theta\right)+i \sin \left(\frac{1}{2} \theta\right)\right)^{2 n}=\cos (n \theta)+i \sin (n \theta) \\
=\cos (n \theta)+\sqrt{\cos ^{2}(n \theta)-1}=T_{n}(2 x)+\sqrt{T_{n}^{2}(2 x)-1}
\end{aligned}
$$

For example, if $n=2$, then $T_{2}(2 x)=8 x^{2}-1$, and so Theorem 3.1 yields (3.1); if $n=3$, then $T_{3}(2 x)=32 x^{3}-6 x$, and Theorem 3.1 yields (3.2). 
After Reznick gave us Theorem 3.1, we learned that T. J. Osler also had observed the connection between Chebyshev polynomials and the problem of simplifying radicals, although Theorem 3.1 is not explicitly given in his paper [10].

In Section 1, we gave one definition of a singular modulus. We offer an alternative definition here. Let ${ }_{2} F_{1}(a, b ; c ; z)$ denote the ordinary hypergeometric series. For each positive rational number $n$, a singular modulus is the unique number $\sqrt{\alpha_{n}}$ satisfying the equation

$$
\frac{{ }_{2} F_{1}\left(\frac{1}{2}, \frac{1}{2} ; 1 ; 1-\alpha_{n}\right)}{{ }_{2} F_{1}\left(\frac{1}{2}, \frac{1}{2} ; 1 ; \alpha_{n}\right)}=\sqrt{n} .
$$

In his second letter to G. H. Hardy [16, p. xxix], [7, p. 60], Ramanujan asserted that

$$
\begin{aligned}
\sqrt{\alpha_{210}}= & (\sqrt{2}-1)^{4}(2-\sqrt{3})^{2}(\sqrt{7}-\sqrt{6})^{4}(8-3 \sqrt{7})^{2} \\
& \times(\sqrt{10}-3)^{4}(4-\sqrt{15})^{4}(\sqrt{15}-\sqrt{14})^{2}(6-\sqrt{35})^{2} .
\end{aligned}
$$

This was first proved by Watson [18] using H. Weber's value for the class invariant $g_{210}[19$, p. 725$]$, where

$$
2 g_{n}^{12}=\frac{1}{\sqrt{\alpha_{n}}}-\sqrt{\alpha_{n}}
$$

and the following remarkable lemma found in Ramanujan's first notebook [15, Vol. 1, p. 320].

LEMma 3.2. Let (as in (3.3))

$$
2 g_{n}^{12}=\frac{1}{\sqrt{\alpha_{n}}}-\sqrt{\alpha_{n}} .
$$

If $g_{n}^{6}=u v$ and

$$
\begin{aligned}
u^{2}+\frac{1}{u^{2}} & =2 U, \quad v^{2}+\frac{1}{v^{2}}=2 V \\
\sqrt{U^{2}+V^{2}-1} & =W, \quad U+V+W+1=2 S
\end{aligned}
$$

then

$$
\begin{aligned}
\alpha_{n}= & \{\sqrt{S}-\sqrt{S-1}\}^{2}\{\sqrt{S-U}-\sqrt{S-U-1}\}^{2} \\
& \times\{\sqrt{S-V}-\sqrt{S-V-1}\}^{2}\{\sqrt{S-W}-\sqrt{S-W-1}\}^{2} .
\end{aligned}
$$

Lemma 3.2 was also proved in Watson's paper but his proof does not shed any light on how Ramanujan might have discovered the formula. A more natural proof is now available [2, pp. 277-280]. The latter proof is based on the simple observation in Lemma 3.3 below.

To obtain elegant representations of $\alpha_{n}$ from Lemma 3.2, we express $g_{n}^{6}$ as a product of two units, for when $n \equiv 2(\bmod 4), g_{n}$ is indeed a unit. Since 
$u$ and $v$ are units, $U, V, W$, and $S$ are algebraic integers. Thus, Lemma 3.2 gives a representation of $\alpha_{n}$ in terms of elegant and relatively simple radicals.

Lemma 3.3. The number

$$
x=(\sqrt{a}-\sqrt{a-1})(\sqrt{b+1}-\sqrt{b})
$$

is a solution of the equation

$$
\frac{1}{x}-x=2(\sqrt{a b}+\sqrt{(b+1)(a-1)}) .
$$

Lemma 3.3 is applicable whenever we encounter an expression involving $x^{-1}-x$. For example, by determining $a$ and $b$ in the expression

$$
\frac{1}{x}-x=\frac{1-2 y^{2}+y^{3}+\sqrt{1-4 y-10 y^{3}-4 y^{5}+y^{6}}}{2 y},
$$

S.-Y. Kang [9, Theorem 3.2, eq. (3.15)] established an explicit formula for the Rogers-Ramanujan continued fraction found in Ramanujan's lost notebook [17, p. 208].

The simple analogue of Lemma 3.3 for the expression $x^{-1}+x$ is given in the next lemma.

Lemma 3.4. The number

$$
x=(\sqrt{a}-\sqrt{a-1})(\sqrt{b}-\sqrt{b-1})
$$

is a solution of the equation

$$
\frac{1}{x}+x=2(\sqrt{a b}+\sqrt{(a-1)(b-1)}) .
$$

The expression $x^{-1}+x$ appears in Ramanujan's evaluations of singular moduli $\alpha_{n}$ when $n$ is divisible by 8 or 16 . Using Lemma 3.4, we now sketch new proofs of two results first proved in [2, p. 285, Theorem 9.5; p. 287, Theorem 9.6].

THEOREM 3.5. If

$$
\alpha_{n}=\frac{1-\sqrt{1-1 / m^{2}}}{2},
$$

then

$$
\begin{aligned}
\alpha_{16 n}= & (\sqrt{m+1}+\sqrt{m})^{8}\{\sqrt{2 m}+1-\sqrt{2 \sqrt{m}(\sqrt{m+1}+\sqrt{2})}\}^{4} \\
& \times\{\sqrt{2 m}-1-\sqrt{2 \sqrt{m}(\sqrt{m+1}-\sqrt{2})}\}^{4} .
\end{aligned}
$$

Proof. From [2, p. 286], it suffices to solve the equation

$$
\begin{aligned}
x^{-1}+x & =2(\sqrt{m+1}+\sqrt{m})^{2}(\sqrt{m}+\sqrt{m-1})^{2} \\
& =2(\sqrt{m+1}+\sqrt{m})^{2}(2 m-1+2 \sqrt{m(m-1)}),
\end{aligned}
$$


where $x=\alpha_{16 n}^{1 / 4}$. Set

$$
\sqrt{a b}=(\sqrt{m+1}+\sqrt{m})^{2}(2 m-1)
$$

and

$$
\sqrt{(a-1)(b-1)}=2(\sqrt{m+1}+\sqrt{m})^{2} \sqrt{m(m-1)} .
$$

Since $(a-1)(b-1)=a b-a-b+1$, we may conclude from (3.6) and (3.7) that

$$
a+b-1=(\sqrt{m}+\sqrt{m+1})^{4}=: s^{2} .
$$

Substituting (3.8) into (3.6), we find that

$$
b\left(s^{2}-b+1\right)=s^{2}(2 m-1)^{2},
$$

which implies that

$$
b=\frac{1+s^{2} \pm 4 s \sqrt{2 m}}{2} .
$$

Since $a$ and $b$ are both solutions of (3.9) and $a \neq b$, we may set

$$
\begin{aligned}
b & =\frac{1+s^{2}+4 s \sqrt{2 m}}{2}=\frac{s}{2}\left(\frac{1}{s}+s+4 \sqrt{2 m}\right) \\
& =s(2 m+1+2 \sqrt{2 m})=s(\sqrt{2 m}+1)^{2} .
\end{aligned}
$$

This implies that the other solution of (3.10) is

$$
a=s(\sqrt{2 m}-1)^{2} .
$$

Substituting (3.11) and (3.12) into Lemma 3.4 yields Theorem 3.5.

To illustrate Theorem 3.5, we find that, for $n=1$ [2, p. 286],

$$
\alpha_{16}=(\sqrt{2}+1)^{4}\left(2^{1 / 4}-1\right)^{8} .
$$

THEOREM 3.6. If

$$
\alpha_{2 n}=(\sqrt{m+1}-\sqrt{m})^{2}(\sqrt{m}-\sqrt{m-1})^{2},
$$

then

$$
\begin{aligned}
\alpha_{8 n}= & \left\{\frac{\sqrt{m}+1+\sqrt{m+1}-\sqrt{(\sqrt{m}+1)(\sqrt{m}+\sqrt{m+1})}\}^{4}}{\sqrt{2}}\right. \\
& \times\left\{\frac{\sqrt{m}-1+\sqrt{m+1}}{\sqrt{2}}-\sqrt{(\sqrt{m}-1)(\sqrt{m}+\sqrt{m+1})}\right\}^{4} .
\end{aligned}
$$

Pr o of. From [2, p. 288], we find that

$$
\frac{1}{\sqrt{x}}+\sqrt{x}=2(\sqrt{m+1}+\sqrt{m})(\sqrt{m}+\sqrt{m-1}),
$$


where $x=\sqrt{\alpha_{8 n}}$. Set

$$
\sqrt{a b}=(\sqrt{m+1}+\sqrt{m}) \sqrt{m}
$$

and

$$
\sqrt{(a-1)(b-1)}=(\sqrt{m+1}+\sqrt{m}) \sqrt{m-1} .
$$

These yield

$$
a=\left(\frac{\sqrt{m}+\sqrt{m+1}+1}{\sqrt{2}}\right)^{2} \quad \text { and } \quad b=\left(\frac{\sqrt{m}+\sqrt{m+1}-1}{\sqrt{2}}\right)^{2}
$$

and the expressions for $\alpha_{8 n}$ then follows from Lemma 3.4. The derivations of $a$ and $b$ from (3.13) and (3.14) are similar to that given in the proof of Theorem 3.5, and we therefore omit the details.

As an illustration of Theorem 3.6, if we set $n=3$, we find that [2, p. 287]

$$
\alpha_{24}=(\sqrt{6+3 \sqrt{3}}-\sqrt{5+3 \sqrt{3}})^{4}(\sqrt{2+\sqrt{3}}-\sqrt{1+\sqrt{3}})^{4} .
$$

We end this section with a simple observation about Lemma 3.2, namely, that it is independent of the definitions of class invariant and singular modulus. Hence, we may restate Lemma 3.2 in the spirit of Lemma 3.3 as

Lemma 3.7. Suppose $x^{-1}-x=2 u v$ where $u$ and $v$ are preferably units in some algebraic number fields. Let

$$
\begin{array}{rlrl}
u+\frac{1}{u} & =2 U, & v+\frac{1}{v}=2 V, \\
\sqrt{U^{2}+V^{2}-1} & =W, \quad U+V+W+1=2 S .
\end{array}
$$

Then

$$
\begin{aligned}
x= & \{\sqrt{S}-\sqrt{S-1}\}\{\sqrt{S-U}-\sqrt{S-U-1}\} \\
& \times\{\sqrt{S-V}-\sqrt{S-V-1}\}\{\sqrt{S-W}-\sqrt{S-W-1}\} .
\end{aligned}
$$

In [8], Chan and S.-S. Huang showed that if

$$
H(q):=\frac{q^{1 / 2}}{1+q}+\frac{q^{2}}{1+q^{3}}+\frac{q^{4}}{1+q^{5}}+\ldots \text {, }
$$

then

$$
\frac{1}{H\left(e^{-\pi \sqrt{n}}\right)}-H\left(e^{-\pi \sqrt{n}}\right)=2 \alpha_{4 n}^{-1 / 4}
$$

Equation (3.15) shows that the Ramanujan-Gordon-Göllnitz continued fraction $H\left(e^{-\pi \sqrt{n}}\right)$ can be evaluated if $\alpha_{4 n}$ is known. Since [4]

$$
\begin{aligned}
& \alpha_{12}^{1 / 4}=(\sqrt{3}-\sqrt{2})(\sqrt{2}-1), \\
& \alpha_{24}^{1 / 4}=(\sqrt{6+3 \sqrt{3}}-\sqrt{5+3 \sqrt{3}})(\sqrt{2+\sqrt{3}}-\sqrt{1+\sqrt{3}})
\end{aligned}
$$


and

$$
\alpha_{28}^{1 / 4}=(3-2 \sqrt{2})(2 \sqrt{2}-\sqrt{7}),
$$

we conclude from Lemma 3.7 that

$$
\begin{aligned}
H\left(e^{-\pi \sqrt{3}}\right)= & \left(\sqrt{\frac{\sqrt{2}+\sqrt{3}+3}{2}}-\sqrt{\frac{\sqrt{2}+\sqrt{3}+1}{2}}\right) \\
& \times\left(\sqrt{\frac{\sqrt{2}-\sqrt{3}+3}{2}}-\sqrt{\frac{\sqrt{2}-\sqrt{3}+1}{2}}\right) \\
& \times\left(\sqrt{\frac{-\sqrt{2}+\sqrt{3}+3}{2}}-\sqrt{\frac{-\sqrt{2}+\sqrt{3}+1}{2}}\right) \\
& \times\left(\sqrt{\frac{\sqrt{2}+\sqrt{3}-1}{2}}-\sqrt{\frac{\sqrt{2}+\sqrt{3}-3}{2}}\right), \\
H\left(e^{-\pi \sqrt{6}}\right)= & \left(\sqrt{\frac{2 \sqrt{2}+\sqrt{6}+\sqrt{3}+3}{2}}-\sqrt{\frac{2 \sqrt{2}+\sqrt{6}+\sqrt{3}+1}{2}}\right) \\
& \times\left(\sqrt{\frac{2 \sqrt{2}+\sqrt{6}-\sqrt{3}}{2}-1}-\sqrt{\frac{2 \sqrt{2}+\sqrt{6}-\sqrt{3}-3}{2}}\right) \\
& \times\left(\sqrt{\frac{\sqrt{2}+\sqrt{3}+3}{2}}-\sqrt{\frac{\sqrt{2}+\sqrt{3}+1}{2}}\right) \\
& \times\left(\sqrt{\frac{-\sqrt{2}+\sqrt{3}+3}{2}}-\sqrt{\frac{-\sqrt{2}+\sqrt{3}+1}{2}}\right), \\
H\left(e^{-\pi \sqrt{7}}\right)= & \times(\sqrt{4+\sqrt{2}-\sqrt{3+\sqrt{2}}})(\sqrt{4-\sqrt{2}-\sqrt{3-\sqrt{2}})} \\
& \times\left(\sqrt{\left.\sqrt{2}+1-2^{1 / 4}\right)\left(2^{1 / 4}-\sqrt{\sqrt{2}-1}\right) .}\right.
\end{aligned}
$$

We have eliminated the details, but in each case the parameters $U, V$, $W$, and $S$ can be routinely calculated.

4. Some radical equalities in the lost notebook. On page 344 in [17], Ramanujan recorded the eight equalities below. Although we have not seen them before, because they are elementary, it is likely that some, or all, of them have been heretofore observed. Because of the notation used by Ramanujan, it might be conjectured that Ramanujan used these equalities in the calculation of class invariants or similar types of numbers. However, we have no examples to substantiate this vague feeling. 
If $g^{4}=5$, then

$$
\frac{\sqrt[5]{3+2 g}-\sqrt[5]{4-4 g}}{\sqrt[5]{3+2 g}+\sqrt[5]{4-4 g}}=2+g+g^{2}+g^{3} .
$$

If $g^{5}=2$, then

$$
\frac{\sqrt{g+3}+\sqrt{5 g-5}}{\sqrt{g+3}-\sqrt{5 g-5}}=g+g^{2}
$$

If $g^{5}=2$, then

$$
\frac{\sqrt{g^{2}+1}+\sqrt{4 g-3}}{\sqrt{g^{2}+1}-\sqrt{4 g-3}}=\frac{1}{5}\left(1+g^{2}+g^{3}+g^{9}\right)^{2} .
$$

If $g^{5}=3$, then

$$
\frac{\sqrt{g^{2}+1}+\sqrt{5 g-5}}{\sqrt{g^{2}+1}-\sqrt{5 g-5}}=\frac{1}{g}+g+g^{2}+g^{3} .
$$

If $g^{5}=2$, then

$$
\sqrt{1+g^{2}}=\frac{1}{\sqrt{5}}\left(g^{4}+g^{3}+g-1\right) .
$$

If $g^{5}=2$, then

$$
\sqrt{4 g-3}=\frac{1}{\sqrt{5}}\left(g^{9}+g^{7}-g^{6}-1\right) .
$$

If $g^{5}=3$, then

$$
\sqrt[3]{2-g^{3}}=\frac{1}{\sqrt[3]{5}}\left(1+g-g^{2}\right)
$$

If $g^{5}=2$, then

$$
\sqrt[5]{1+g+g^{3}}=\frac{1}{\sqrt[10]{5}} \sqrt{1+g^{2}}
$$
that

Equalities (4.1)-(4.4) are readily proved if we use the elementary fact

$$
\frac{A+B}{A-B}=M \quad \text { if and only if } \quad(M-1) A=(M+1) B .
$$

As an illustration, we prove (4.1).

If $A=\sqrt[5]{2 g+3}$ and $B=\sqrt[5]{4 g-4}$, by (4.9), it suffices to prove that

$$
\left(1+g+g^{2}+g^{3}\right) \sqrt[5]{2 g+3}=\left(3+g+g^{2}+g^{3}\right) \sqrt[5]{4 g-4} .
$$

Since $g^{4}=5$, we have

$$
1+g+g^{2}+g^{3}=\frac{g^{4}-1}{g-1}=\frac{4}{g-1}
$$


and

$$
3+g+g^{2}+g^{3}=2+\frac{4}{g-1}=\frac{2 g+2}{g-1} .
$$

Thus, (4.10) is equivalent to the equality

$$
2 \sqrt[5]{2 g+3}=(g+1) \sqrt[5]{4 g-4},
$$

or

$$
8(2 g+3)=(g+1)^{5}(g-1),
$$

which is readily verified by again using the hypothesis $g^{4}=5$.

Equalities (4.5)-(4.8) can easily be established by raising each side to an appropriate power.

Next to (4.7), Ramanujan wrote " $g=3$ ". Indeed, it is readily verified that $g=3$ is also a root of (4.7).

To the right of (4.8), Ramanujan wrote

$$
g^{5}+5 g^{3}+5 g+2=0 .
$$

Indeed, from (4.8),

$$
0=g^{10}+5 g^{8}+5 g^{6}-10 g^{3}-10 g-4=\left(g^{5}-2\right)\left(g^{5}+5 g^{3}+5 g+2\right),
$$

which proves (4.11).

We are very grateful to the referee for pointing out several inaccuracies in our original manuscript.

\section{References}

[1] B. C. Berndt, Ramanujan's Notebooks, Part IV, Springer, New York, 1994.

[2] -, Ramanujan's Notebooks, Part V, Springer, New York, 1998.

[3] B. C. Berndt, H. H. Chan and L.-C. Zhang, Ramanujan's class invariants and cubic continued fraction, Acta Arith. 73 (1995), 67-85.

[4] —, 一, 一, Ramanujan's singular moduli, Ramanujan J. 1 (1997), 53-74.

[5] - - - -, Ramanujan's class invariants, Kronecker's limit formula, and modular equations, Trans. Amer. Math. Soc. 349 (1997), 2125-2173.

[6] - - - - Ramanujan's association with radicals in India, Amer. Math. Monthly 104 (1997), 913-919.

[7] B. C. Berndt and R. A. Rankin, Ramanujan: Letters and Commentary, Amer. Math. Soc., Providence, 1995; London Math. Soc., London, 1995.

[8] H. H. Chan and S.-S. Huang, On the Ramanujan-Gordon-Göllnitz continued fraction, Ramanujan J. 1 (1997), 75-90.

[9] S.-Y. Kang, Ramanujan's formulas for the explicit evaluation of the Rogers-Ramanujan continued fraction and theta-functions, Acta Arith., to appear.

[10] T. J. Osler, Cardan polynomials and the reduction of radicals, preprint.

[11] S. Ramanujan, Question 525, solution by N. S. Aiyar, J. Indian Math. Soc. 6 (1914), 191-192.

[12] -, Question 682, solution by "Zero", ibid. 7 (1915), 160. 
[13] S. Ramanujan, Question 1070, solutions by S. D. Chowla, N. B. Mitra, and S. V. Venkataraya Sastri, ibid. 11 (1919), 160.

[14] -, Question 1076, ibid. 11 (1919), 199.

[15] —, Notebooks (2 volumes), Tata Institute of Fundamental Research, Bombay, 1957.

[16] - Collected Papers, Chelsea, New York, 1962.

[17] —, The Lost Notebook and Other Unpublished Papers, Narosa, New Delhi, 1988.

[18] G. N. Watson, Theorems stated by Ramanujan (XII): A singular modulus, J. London Math. Soc. 6 (1931), 65-70.

[19] H. Weber, Lehrbuch der Algebra, dritter Band, Chelsea, New York, 1961.

[20] L.-C. Zhang, Ramanujan's class invariants, Kronecker's limit formula and modular equations (II), in: Analytic Number Theory, Proc. Conf. in Honor of Heini Halberstam, Vol. 2, B. C. Berndt, H. G. Diamond, and A. J. Hildebrand (eds.), Birkhäuser, Boston, 1996, 817-838.

Department of Mathematics

University of Illinois

1409 West Green Street

Urbana, Illinois 61801

U.S.A.

E-mail: berndt@math.uiuc.edu

Department of Mathematics

Southwest Missouri State University

Springfield, Missouri 65804

U.S.A.

E-mail: liz917f@mail.smsu.edu
Department of Mathematics National University of Singapore

Kent Ridge Singapore 119260 Republic of Singapore E-mail: chanhh@math.nus.edu.sg

Received on 27.1.1998

and in revised form on 15.6.1998 